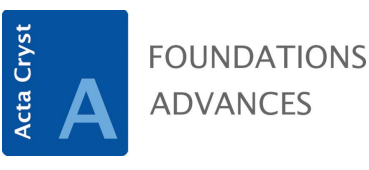

ISSN 2053-2733

\section{Space Group Visualizer. By Eckhard Hitzer and Christian Perwass. Independently published, 2021. Paperback, pp. 162. Price EUR 22.25, USD 24.99. ISBN 979-8719838618.}

\author{
Ulrich Müller*
}

Fachbereich Chemie, Universität Marburg, Hans-Meerwein-Strasse, Marburg, 35032, Germany. *Correspondence e-mail: mueller@chemie.uni-marburg.de

Keywords: space-group theory; crystallographic software.

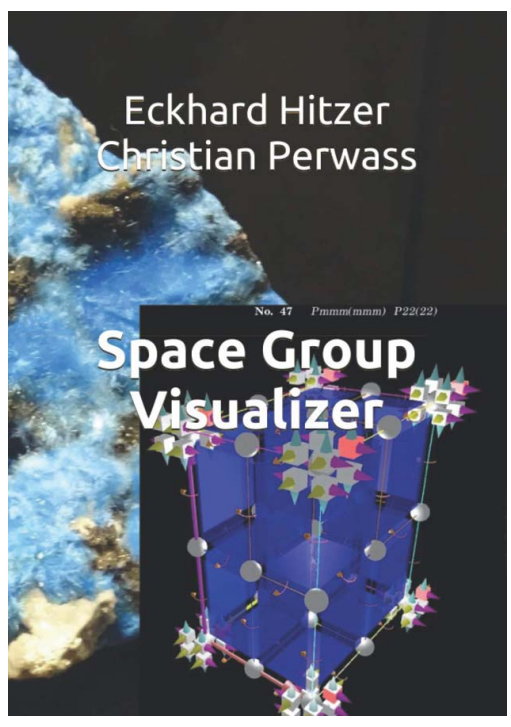

The text refers to the computer program Space Group Visualizer that can be downloaded from http://spacegroup.info/. It is a program that interactively displays the symmetry elements of one unit cell of a chosen space group. The view can be rotated on the screen, which is helpful for people who find it difficult to imagine the spatial arrangement. By choosing a symmetry element and positioning the mouse arrow on the screen, arrows or small figures move back and forth, simulating the execution of the symmetry operation. For higher symmetries, the images on the screen are overcrowded with planes, lines, arrows and little figures of cubes with cones. It is not possible to insert atoms and to display crystal structures. Here we do not discuss the details of the software, but the contents of the book.

The first cursory impression is that this is a textbook or a tutorial for the computer program. In fact, Chapters 6,8 and 10 and the appendix (a total of 64 pages) are such a tutorial for the Space Group Visualizer. The rest of the book is not a textbook, but consists of chapters that are reprints of published and unpublished journal articles and lecture notes. There is no uniform book style, no gradual development of notions in the sequence of the chapters and there are frequent repetitions in different chapters.

For the user of the software it is irrelevant on what mathematical basis and procedures the visualization program has been programmed. For someone who wants to use the program to 'see' the symmetry of a space group, the book is unimportant. The tutorial in the appendix is just a copy of the tutorial that comes with the software.

However, for those interested in the mathematical background of the programming, the book gives insights. The calculations do not follow the usual crystallographic procedures using coordinate triplets and matrix calculus. It is rather a new approach for the mathematical treatment of space groups applying geometric algebra. Geometric algebra is based on vector multiplications and is explained in Chapter 2 and three more times in Chapters 3,5 and 7. However, the meaning of the formulations remains unclear until Chapter 5 (page 39). The unintelligibility is also due to a lack of uniformity in the used symbols. In some chapters, a vector is simply designated by an italic letter, say $c$, in other chapters it is $\vec{c}$, sometimes it is a roman c, sometimes a bold face $\mathbf{c}$ or $\mathbf{c}$, sometimes a bold-face italic $\boldsymbol{c}$. The letters $a, b, c, \ldots$ and $\boldsymbol{a}, \boldsymbol{b}, \boldsymbol{c}, \ldots$ are also used to designate reflections. Sometimes unexplained symbols appear, e.g. $C I(4,1)$ on page 23 . Only crystallographers with a sound mathematical background will be able to follow the reasoning; 'normal' crystallographers, who often are chemists or biochemists, will find it more difficult.

The most used kind of vector multiplication is called a 'vector product' or 'geometric product' (these seem to be synonyms). A 'geometric product' is formulated in such a way that it is not recognizable as such: $a b$ or $\vec{a} \vec{b}$. It is neither a dot nor a cross product, but a combination of two products, $\vec{a} \vec{b}=\vec{a} \cdot \vec{b}+\vec{a} \wedge \vec{b}$, where $\vec{a} \cdot \vec{b}$ is the scalar (dot) product and $\vec{a} \wedge \vec{b}=|\vec{a}||\vec{b}| \mathbf{i} \sin \alpha$ means 'wedge product' (i = unit area element in the plane spanned by $\vec{a}$ and $\vec{b}, \alpha=$ angle between $\vec{a}$ and $\vec{b}$ ). The 'geometric product' thus is a scalar. The reflection of a vector $\vec{x}$ at a plane perpendicular to $\vec{a}$ is then formulated as $\vec{x}^{\prime}=-\vec{a}^{-1} \vec{x} \vec{a}$. A sequence of two geometric products generates a rotation of $\vec{x}$, and additional geometric products result in further symmetry operations. 
The use of this vector algebra makes it possible to derive all crystallographic space groups. Allegedly, this is independent of crystallographic coordinates; but that is not quite true, because the crystallographic basis vectors are needed to define the bases for the vectors. The chosen vector bases deviate from the crystallographic ones; for example, for the cubic system the chosen basis is $\vec{a}=\frac{1}{2} \mathbf{a}, \vec{b}=\frac{1}{2}(\mathbf{a}+\mathbf{b}), \vec{c}=\frac{1}{2}(\mathbf{a}+\mathbf{c}), \mathbf{a}, \mathbf{b}, \mathbf{c}$ being the crystallographic basis vectors. A starting point are three 'physical crystal vectors'; these seem to be vectors between symmetry-equivalent points in a space group (including the crystallographic basis vectors), but that is not explained and not quite clear.

The terminology deviates in parts from the usual crystallographic terminology. For example, a unit cell is called a crystal cell and a primitive unit cell is called an elementary cell (and thus is something different from the German Elementarzelle, which means unit cell). The terms 'basis vector' and 'crystallographic basis' do not occur. Rotoinversions are called rotary inversions, and it is never mentioned that a rotoinversion is always also a rotoreflection. New symbols are introduced for space groups and point groups, for example, $P 6_{c} 2$ instead of $P 6_{3} / m m c, P 2 \overline{2}$ instead of $P 2 / m$ and $2 \underline{2}$ instead of
$2 / m$. For a crystallographer that is a confusing inconvenience. One chapter deals with the conversion of this 'geometric algebra versor notation' to Hermann-Mauguin symbols, a term that is never mentioned. No distinction is made between space group and space-group type.

Chapter 9, 'The hidden beauty of gold', is completely superfluous. Every crystallographer is familiar with the simple face-centred cubic crystal structure of gold and does not need such a long-winded and complex description with so many confusing images.

The typography of the book is non-uniform and does not meet typographic standards. In some chapters there are up to 90 characters per line, while the normal standard is a maximum of 75 , preferably 65 ; that hampers reading. There are no running titles and the numbering of the sections does not include the chapter numbers. The book has no subject index and no list of symbols.

In summary, the software is nice for people who lack spatial imagination and who want to have a three-dimensional impression of the arrangement of symmetry elements in a space group. The book, however, is not useful for crystallographers. 